Canadian

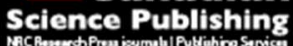

Canadian Journal of Microbiology Revue canadienne de de microbiologie

\title{
Investigating the expression of F10 and G11 xylanases from Aspergillus niger A09 with qPCR
}

\begin{tabular}{|r|l|}
\hline Journal: & Canadian Journal of Microbiology \\
\hline Manuscript ID & cjm-2015-0394.R4 \\
\hline Danuscript Type: & Article \\
\hline Complete List of Authors: & $\begin{array}{l}\text { Cui, Shixiu; Henan Agricultural University, College of Life Sciences } \\
\text { Wang, Tianwen; East China University of Science and Technology, School } \\
\text { of Biotechnology } \\
\text { Hu, Hong; Henan Academy of Sciences, Institute of Biology Co., Ltd } \\
\text { Liu, Liangwei; Henan Agricultural University, College of Life Sciences } \\
\text { Song, An-Dong; Henan Agricultural University, College of Life Sciences } \\
\text { Chen, Hongge; Henan Agricultural University, College of Life Sciences }\end{array}$ \\
\hline Keyword: & \begin{tabular}{l} 
Aspergillus niger, qPCR, F10 xylanase, G11 xylanase, Expression pattern \\
\hline
\end{tabular} \\
\hline
\end{tabular}


1 Investigating the expression of F10 and G11 xylanases in

2 Aspergillus niger A09 with qPCR

3

4 Shixiu Cui ${ }^{1 \#}$, Tianwen Wang²\#, Hong Hu${ }^{3}$, Liangwei Liu ${ }^{1}$, 5 Andong Song ${ }^{1}$, Hongge Chen ${ }^{1 *}$

6 1. College of Life Sciences, Henan Agricultural University,

7 Zhengzhou, 450002, P. R. China

8 2. School of Biotechnology, State Key Laboratory of Bioreactor

9 Engineering, East China University of Science and Technology,

10 Shanghai, 200237, P. R. China

11 3. Institute of Biology Co., Ltd, Henan Academy of Sciences,

12 Zhengzhou, 450008, P. R. China

13 \#Contributed equally to this work.

*Corresponding author, E-mail: honggeyz@163.com 

62 ${ }_{66}^{66}$

\section{Abstract}

There exist significant differences between the two main types of xylanase, family F10 and G11. A clear understanding of the expression pattern of microbial F10 and G11 under different culture conditions would facilitate better production and industrial application of xylanase. In this study, the fungal xylanase producer Aspergillus niger A09 was systematically investigated in terms of induced expression of xylanase F10 and G11. Results showed that carbon and nitrogen source could influence xylanase F10 and G11 transcript abundance, with G11 more susceptible to changes in culture media composition. The most favorable carbon and nitrogen source for high G11 and low F10 production by $A$. niger A09 were xylan $(2 \%)$ and $\left(\mathrm{NH}_{4}\right)_{2} \mathrm{C}_{2} \mathrm{O}_{4}$ $(0.3 \%)$, respectively. Following cultivation at $33^{\circ} \mathrm{C}$ for $60 \mathrm{~h}$, the highest xylanase activity (1132 IU per gram of wet mycelia) was observed. Based on differential gene expression of F10 and G11, as well as their different properties, we deduced that the F10 protein initially targeted xylan and hydrolyzed it into fragments including xylose, after which xylose acted as the inducer of F10 and G11 gene expression. These speculations also accounted for our failure to identify conditions favoring the high production of F10, but a low production of G11.

Key words: Aspergillus niger, qPCR, F10 xylanase, G11 xylanase, Expression pattern 每 ${ }^{4}$ 65 


\section{Introduction}

Xylan is the second most abundant polysaccharide on Earth. As an integral component of the plant cell wall, xylan accounts for roughly $1 / 3$ of all carbohydrate in the plant, or $7-35 \%$ of the dry cell weight (Deutschmann and Dekker 2012). Xylanases are a group of enzymes that can efficiently degrade xylan to (oligo-) xylose (Ahmed et al. 2009; Adsul et al. 2009). Xylanases can be used as biocatalysts in the production of biofuels from plant biomass (Harris et al. 2014), in animal feed production (Olukosi et al. 2007), in preparation of functional food xylo-oligosaccharides (Wang et al. 2015) and in the paper and pulp industry (Buchert et al. 1994).

Due to the structural heterogeneity of xylan, the complete degradation of xylan is usually orchestrated through the function of different xylanases, including endo- $\beta-1,4-x y l a n a s e$, $\beta$-xylosidase, acetyl esterase, arabinosidase, etc. (Lee et al. 2003). However, endo- $\beta$-1,4-xylanase (EC 3.2.1.8, hereinafter referred to as xylanase) is responsible for cleaving beta-1,4-glycosidic bonds within the xylan backbone and plays a critical role in xylan degradation.

The biochemical properties of xylanase are highly dependent on their structure and origin. Based on amino acid sequence similarity and hydrophobic cluster analysis, the vast majority of xylanases are classified into glycoside hydrolase family 10 and 11, corresponding to the formerly named family $\mathrm{F}$ and family $\mathrm{G}$, respectively (Henrissat 1991; Henrissat and Bairoch 1993). F10 xylanases have a high molecular weight (>30 kDa) and a low $\mathrm{pI}$ value, with a typical 8 -fold $\alpha / \beta$ barrel structure. In contrast, G11 xylanases have a low molecular weight $(<30 \mathrm{kDa})$ and a high $\mathrm{pI}$ value, with a $\beta$-jelly roll fold structure. The notable difference between the two families are their final hydrolysis products: F10 
100

101

102

103

104

105

106

107

108

109

110

111

112

113

114

115

116

117

118

119

120

121

122

123

124

125

126

127

128

129

130

131

132

xylanases release smaller oligosaccharides with more xylose, while G11 xylanases release larger oligosaccharides with nearly no xylose. This is due to the fact that the F10 xylanase has substrate binding sites smaller than those of G11 (four to five for F10 while at least seven for G11, as stated by Collins et al. (2005)), leading to the F10's greater catalytic versatility, or lower substrate specificity than G11 (Jeffries 1996; Biely et al. 1997). In fact, F10 xylanases also exhibit activity on short xylo-oligosaccharides, as well as on low molecular mass cellulose substrate, the activity compatible with $\beta$-xylosidase and cellulase, respectively (Collins et al. 2005). The property differences between F10 and G11 make them a functionally complementary pair for xylanase-producing microbes to digest xylan efficiently.

However, as far as the application of xylanases are concerned, it is conceivable that one type of xylanase may be more desirable than the other. For example, the G11 xylanase should be more advantageous for xylo-oligosaccharides production than an F10 xylanase, since the F10 xylanase releases too much xylose, a side product which will increase the cost of removing xylo-oligosaccharides with degree of polymerization (DP) 2-7. That is probably why most work on xylo-oligosaccharide production made use of G11 xylanases (Liu et al. 2008; Bragatto et al. 2013; Wang et al. 2015; Qu et al. 2015). On the other hand, when considering biofuel production from biomass, an F10 xylanase is more suitable for thorough biomass degradation than a G11 xylanase. Although the production of a desirable type of xylanase by heterogeneous expression in either Escherichia coli or Pichia pastoris is now technically feasible, xylanase production by the natural xylan-utilizing fungus Aspergillus niger is still an attractive option. Considered a generally-regarded as safe (GRAS) fungus (Schuster et al. 2002), 
133 A. niger possesses a high protein secretion ability (de Oliveira et al. 2011), the appropriate glycosylation required for enzyme function (Han and Lei, 1999), and the rich enzyme complex (Kniemeyer 2011). Therefore it is regarded as an ideal source for exploiting xylanase as a catalyst. Currently, little has been reported on gene expression patterns of $A$. niger F10 and G11 xylanases in response to different carbon and nitrogen sources. Elucidating this may provide the possibility of selectively producing either F10 or G11 simply by adjusting the culture media components, making the production of a desirable xylanase preparation cost-effective.

We previously noted that the high xylanase producing strain $A$. niger A09 produced two visible xylanase bands on SDS-PAGE zymogram analysis; one identified as an F10 xylanase and the other a G11. This strain provides an excellent source to investigate the formation pattern of F10 and G11. In this work, the expression of xylanase F10 and G11 from A. niger A09 were systematically investigated using $\mathrm{qPCR}$ and other related methods in the hope of clarifying the expression mechanisms of F10 and G11, and also to optimize culture media composition to allow for the selective production of either F10 or G11 xylanases.

\section{Materials and Methods}

\section{Strain and primers}

The $A$. niger A09 strain was a lab stock copy of a previously screened sample submitted to the China General Microbiological Culture Collection Center. Primers used in the study are listed in Table 1. These primers were designed based on the sequences found in Genbank (18S rRNA, xylanase G11 (DQ147775) and F10 (XM_001389959.2)) with the aid of Beacon 
Designer software by Premier Biosoft (Palo Alto, USA). All primers were synthesized by the Sangon Company (Shanghai, China).

\section{Chemicals}

Birchwood xylan substrate, DEPC solution, and EDTA were products of Sigma (St. Louis, MO, USA). Components (peptone, yeast extract) for medium preparation were from Oxiod (Hampshire, UK). RNAiso ${ }^{\mathrm{TM}}$ RNA isolation kit, Real time RT-PCR kit, and protein marker were bought from Takara (Dalian, China). Other chemicals were of analytical grade from local suppliers. Development solution 3,5-dinitrosalicylic acid (DNS) was a fresh preparation in each assay.

\section{Culture medium}

Culture medium PDA (slants or plates) was prepared with fresh potato. PDA was also used for preservation of seed culture.

Medium for enzyme production was prepared by supplementing the selected carbon source and nitrogen source to the basic medium (carbon source, 2; ammonium oxalate, 0.3; $\mathrm{K}_{2} \mathrm{HPO}_{4} \cdot 3 \mathrm{H}_{2} \mathrm{O}, 0.1 ; \mathrm{MgSO}_{4} \cdot 7 \mathrm{H}_{2} \mathrm{O}, 0.05 ; \mathrm{KCl}, 0.05 ; \mathrm{FeSO}_{4} \cdot 7 \mathrm{H}_{2} \mathrm{O}$, $0.001, \mathrm{w} \%$,). Carbon source was selected from xylan, xylose, glucose, maltose, cellobiose, arabinose, lactose; and nitrogen source from yeast extract, $\mathrm{NH}_{4} \mathrm{NO}_{3},\left(\mathrm{NH}_{4}\right)_{2} \mathrm{SO}_{4}$, peptone, urea, and $\mathrm{NaNO}_{3}$, besides ammonium oxalate.

\section{Preparation of single-spore suspension}

The spores from 5-6 day's cultivation on a PDA slant were aseptically transferred to autoclaved flasks with an appropriate volume of distilled water and suitable amount of glass beads. 
The flasks were then shaken on a shaker at $220 \mathrm{rpm}$ for about 1 $\mathrm{h}\left(33^{\circ} \mathrm{C}\right)$ to completely disperse the spores (Ho and Ko 1997) and create a spore suspension.

\section{Cultivation of $A$. niger and preparation of enzyme extract}

The spore suspension $(1 \%, \mathrm{v} / \mathrm{v})$ was inoculated into $50 \mathrm{~mL}$ of sterilized liquid medium in $250 \mathrm{~mL}$ flasks in triplicate. Cultivation was carried out at $33^{\circ} \mathrm{C}$ and $220 \mathrm{rpm}$. Following growth, the filtrate of the culture broth was collected after passage through 4-layered cheesecloth, and centrifuged at 4200 rpm for $10 \mathrm{~min}\left(4^{\circ} \mathrm{C}\right)$. The supernatant was used as enzyme extract. Collected mycelium was subjected to biomass analysis or RNA extraction after sufficient washing with distilled water.

\section{Enzymatic assay}

To $0.1 \mathrm{~mL}$ solution of Birchwood xylan (1\%, in $0.2 \mathrm{M} \mathrm{HAc}-\mathrm{NaAc}$ buffer, pH4.6), $0.1 \mathrm{~mL}$ appropriately diluted enzyme extract was added and thoroughly mixed. The reaction was kept at $50^{\circ} \mathrm{C}$ for $15 \mathrm{~min}$ and stopped by the addition of $0.6 \mathrm{~mL}$ DNS solution. The mixture was then boiled for 10 min, after which distilled water was added to a final volume of $5 \mathrm{~mL}$, and the optical density at $550 \mathrm{~nm}$ recorded to calculate the amount of reducing sugar (as xylose) generated. Pre-inactivated enzyme was used as a control. One unit was the amount of enzyme required for the generation of $1 \mu \mathrm{mol}$ xylose in $1 \mathrm{~min}$ under the conditions specified above. The calculation was done with the equation $\mathrm{E}=1.53 \mathrm{D} \times \mathrm{OD}_{550}$, where $\mathrm{E}$ was the activity (U), D was the dilution, and $\mathrm{OD}_{550}$ was the optical density from the spectrometer (Gusakov et al. 2011; Rivers et al. 1984).

\section{Zymographic analysis}


In zymographic analysis (Leber and Balkwill 1997), proteins in the sample were denatured, separated by SDS-PAGE, and renatured to catalyze the substrate evenly dispersed in the gel (Chen and Buller 1995). Briefly, xylan (1\%) dissolved in 0.1 $\mathrm{mol} / \mathrm{L} \mathrm{NaOH}$ was added to a resolving gel (1/10, v/v). The $\mathrm{pH}$ was adjusted to 8.8 with $6 \mathrm{~mol} / \mathrm{L} \mathrm{HCl}$ before the addition of tetramethylethylenediamine (TEMED) and ammonium persulfate (APS). The enzyme extract was mixed with loading buffer and boiled for $10 \mathrm{~min}$ at $100^{\circ} \mathrm{C}$. After centrifugation, the supernatant was loaded onto the gel for SDS-PAGE. Following SDS-PAGE, the gel was washed for 60 min with $2.5 \%$ Trixton-X100 to remove SDS, and the gel incubated in $50 \mathrm{mM}$ $\mathrm{HAc}-\mathrm{NaAc}$ buffer for $30 \mathrm{~min}\left(50^{\circ} \mathrm{C}\right)$. The reaction was stopped by placing the gel at $4^{\circ} \mathrm{C}$. Gel staining was done by incubating the gel with $1 \mathrm{mg} / \mathrm{ml}$ Congo red for $20 \mathrm{~min}$ with gentle shaking. De-staining was performed in $1 \mathrm{M} \mathrm{NaCl}$ until colorless or light yellow bands were visible in the dark-red background, after which $1 \mathrm{M} \mathrm{HCl}$ was added to change the background color into deep blue.

\section{The transcriptional analysis}

Collected mycelium were ground to a powder and used for RNA isolation, following manufacturer's instructions. The concentration and purity of isolated total RNA were analyzed by measuring the $\mathrm{OD}_{260}$ and $\mathrm{OD}_{280}$ of 50-fold dilution with DEPC-containing water (also as a blank). The integrity of isolated RNA was checked by agarose gel electrophoresis with gel concentration of $1 \%$. For each lane, $0.8 \mu \mathrm{g}$ of total RNA was loaded. Total cDNA was obtained by reverse transcription of the isolated total RNA. The mixture for cDNA synthesis can be found in Table $\mathrm{S} 1$. Reverse-transcription was carried out at $37^{\circ} \mathrm{C}$ for $15 \mathrm{~min}$, and 
265

266

then the sample was incubated at $85^{\circ} \mathrm{C}$ for $5 \mathrm{~s}$ to inactivate the reverse transcriptase (Wacker and Godard 2005). The semi-quantitative RT-PCR was carried out under the conditions in Table S2 and Table S3. In the real-time PCR, the reaction system specified in Table S4 was used, and the 18S rRNA gene was used as the reference. For each group, three samples were used: \#1 was the calibration, the other two were tests. The data was analyzed with $2^{-\Delta \Delta C_{t}}$ method (Schmittgen and Livak 2008).

\section{Results}

The effect of different carbon and nitrogen sources on mycelium growth and xylanase activity of $A$. niger

We determined xylanase activity and mycelium fresh weight at two typical time points (40h and 72h) during culture of $A$. niger A09 on different carbon sources (Table 2). At each time point, the ratios of activity to mycelium fresh weight were also calculated as an indicator parameter for easier comparison.

From these data, we could see that the tested carbon sources could be utilized differently by $A$. niger A09. Lactose and arabinose couldn't support the growth of this fungus, indicating the lack of corresponding enzymes to efficiently metabolize these two carbon sources. Maltose and glucose were comparable in supporting growth, but were not suitable carbon sources for enzyme production. Cellobiose was a good carbon source for fast mycelium growth, since the cell weight reached a high value at $40 \mathrm{~h}$, comparable to that of $72 \mathrm{~h}$. Regarding xylanase, there was a negligible change during this course. Xylan and xylose were better carbon sources for the growth and enzyme production of A.niger A09. Xylan was a better carbon source for quick and sustainable accumulation of biomass, although the performance 
on xylose was also acceptable. Xylan was also a better choice as carbon source for xylanase production, because a higher activity (15.3 IU/ml after 40h) could be achieved. These results suggested that, for the induced expression of xylanase, xylan was better than the monosaccharide xylose, implying that the required additional steps prior to metabolizing xylan didn't constitute a problem limiting the efficient utilization of xylan by A. niger A09.

Using xylan as the carbon source, the influence of different nitrogen sources on mycelium accumulation and xylanase secretion was shown in Table 3. Preferences for nitrogen sources were observed, and three nitrogen sources (urea, peptone, $\mathrm{NaNO}_{3}$ ) couldn't be utilized by this fungus (data not shown). Of the four utilizable nitrogen sources (yeast extract, $\left(\mathrm{NH}_{4}\right)_{2} \mathrm{SO}_{4}$, $\left(\mathrm{NH}_{4}\right)_{2} \mathrm{C}_{2} \mathrm{O}_{4}, \mathrm{NH}_{4} \mathrm{NO}_{3}$, better xylanase specific activity at extended culture time were observed with the latter three nitrogen sources. Interestingly, for most microbes, yeast extract serves as a good nutrient source for their growth. Therefore, the decrease in specific activity should be ascribed to the continued accumulation of mycelium, leading to a decreased ratio in specific activity.

\section{Semi-quantitative analysis of the influence of carbon sources and nitrogen sources on transcription of F10 and G11}

As shown in the previous experiment, A.niger A09 couldn't grow with lactose or arabinose as the carbon source. Therefore, xylan, xylose, glucose, cellobiose, and maltose were used as individual carbon sources to compare xylanase transcription level by semi-quantitative PCR analysis. With the $18 \mathrm{~S}$ rRNA gene as a reference, the amounts of cDNA template for different samples were determined to get the equal quantity of reference gene 
331

332

333

334

335

PCR products, and the appropriate cycling number was identified to ensure PCR reactions within the exponential amplification phase. Using the determined cDNA template amounts and cycling number, F10 and G11 transcripts were correspondingly amplified. As shown in Fig. S1, the relatively high transcription of G11was found with xylan and xylose as the carbon source. A similar phenomenon was also observed in the case of F10, although the transcription level on these two carbon sources were slightly lower than that of G11. For G11, variation in transcription levels were more significant. However, F10 exhibited more stable and lower transcription levels with all five carbon sources tested. When the $40 \mathrm{~h}$ and $72 \mathrm{~h}$ samples collected from the four different nitrogen sources $\left[\left(\mathrm{NH}_{4}\right)_{2} \mathrm{C}_{2} \mathrm{O}_{4},\left(\mathrm{NH}_{4}\right)_{2} \mathrm{SO}_{4}\right.$, yeast extract, $\mathrm{NH}_{4} \mathrm{NO}_{3}$ ] were analyzed, G11 transcription levels were higher than those of F10, however, the semi-quantitative PCR failed to reveal meaningful differences in expression level between samples (Fig. S2).

\section{Effect of carbon sources on transcriptional level revealed by real-time PCR analysis}

Primers are crucial for real-time PCR reliability. A melting curve analysis of $18 \mathrm{~S}$ rRNA, F10, and G11 gene products (Fig. S3) showed that the melting curve for each product had a single peak, indicating that the primer pairs used were specific for the targeted genes. The real-time PCR (Fig.1, Table S5) confirmed that the transcription levels of F10 and G11 were influenced, to different extents, by the carbon sources used in the medium. Using the transcription level of F10 xylanase at 40h on glucose medium as a reference, we found that, at 40h, the F10 transcription levels were relatively high with xylose and maltose as carbon source. However, when xylan and cellobiose were used, the transcription levels were rather low. When the 
cultivation time reached $72 \mathrm{~h}$, the superiority of xylan as a carbon source was more prominent: with an approximate 6.5-fold increase observed for F10. In contrast, with xylose, a 2.7-fold decrease in transcript abundance was observed. Moreover, a similar trend was also observed with maltose and cellobiose. These results together indicated that the transcription of F10 xylanase was significantly dependent on the induction by the available carbon source. Sugars like glucose, xylose, and maltose were better carbon sources that supported increased F10 transcription levels at an earlier time (40h). These observations may reflect a greater ease of A.niger A09 in metabolizing these carbon sources. A continued increase in transcript abundance was only seen with xylan and cellobiose, although the F10 transcription level with cellobiose was much lower than that with glucose. When the G11 xylanase was considered, higher transcription levels were found with xylan and xylose as carbon source, which was the same result as revealed by semi-quantitative PCR analysis. In the case of cellobiose at $40 \mathrm{~h}$ and that of maltose at $72 \mathrm{~h}$, the G11 transcription levels were much lower versus the reference. While the G11 transcription level on xylose almost remained the same from $40 \mathrm{~h}$ to $72 \mathrm{~h}$, there was a sharp increase in G11 transcripts on xylan from $40 \mathrm{~h}$ to $72 \mathrm{~h}$. Therefore, in subsequent experiments, xylan was chosen as the carbon source.

\section{Effect of nitrogen source on transcription level revealed by real-time PCR analysis}

Using xylan as the carbon source, seven different nitrogen sources were tested (yeast extract, ammonium oxalate, $\mathrm{NH}_{4} \mathrm{NO}_{3}$, $\left(\mathrm{NH}_{4}\right)_{2} \mathrm{SO}_{4}$, peptone, urea, and $\left.\mathrm{NaNO}_{3}\right)$. Unfortunately, peptone, urea and $\mathrm{NaNO}_{3}$ could not support the fungus to accumulate sufficient sample for analysis. Therefore, only the yeast extract, 
397

398

399

400

401

402

403

404

405

406

407

408

409

410

411

412

413

414

415

416

417

418

419

420

421

422

423

424

425

426

427

428

429

ammonium oxalate, $\left(\mathrm{NH}_{4}\right)_{2} \mathrm{SO}_{4}$, and $\mathrm{NH}_{4} \mathrm{NO}_{3}$ were subsequently investigated. As shown in Figure 2 and Table S6, except for the yeast extract, all the other utilizable nitrogen sources exhibited an increase in the G11 transcription level with the extended cultivation time. Ammonium oxalate yielded the highest G11 transcription level. A possible reason for this phenomenon was that the fungus could utilize both the nitrogen and carbon source contained within ammonium oxalate.

\section{Analysis of samples collected at different time points with real-time PCR}

Based on the transcription analyses, we decided to use xylan and ammonium oxalate as the optimal carbon and nitrogen source, respectively. An analysis of F10 and G11 transcription levels was carried out during the cultivation process over $24 \mathrm{~h}$ to $84 \mathrm{~h}$. At the same time, the weight of mycelium, xylanase activity, and specific activity were also determined (Fig. 3). The weight of mycelium reached a maximum at $84 \mathrm{~h}$, while both the xylanase activity and specific activity reached their highest value at $60 \mathrm{~h}$. Extended culture time ( $84 \mathrm{~h}$ ) was beneficial for the accumulation of mycelium; however, it didn't yield a higher xylanase activity. Since specific activity was the ratio of activity to mycelium weight, a significant decrease in specific activity was consequently observed. From Fig. 4, we could clearly see that the transcription level of G11 was much higher than that of F10 at each analyzed time point (Table S7). Zymographic analysis of samples collected at different time points showed that both F10 and G11 enzyme increased with extended time (Fig. S4). However, the G11 activity band was not as dominant as the transcriptional data observed, hinting that the specific activity of G11 might be lower than that of F10. It might also indicate the translation of G11 transcripts was not as efficient as that of 
430

431

432

433

434

435

436

437

438

439

440

441

442

443

444

445

446

447

448

449

450

451

452

453

454

455

456

457

458

459

460

461

462

F10 transcripts.

\section{Discussion}

Nutrients are known to affect the expression of certain

proteins/enzymes (de Souza et al. 2013). Our initial intent was

to identify cultivation conditions favoring the efficient accumulation of either F10 or G11 xylanase. In this study, we systematically investigated the influence of carbon and nitrogen sources on the transcript abundance of the two main types of xylanase, F10 and G11 from A. niger A09. Although we failed in finding a condition that favored the high production of F10 and low production of G11, we successfully found that, by $60 \mathrm{~h}$ of cultivation (xylan, $\left(\mathrm{NH}_{4}\right)_{2} \mathrm{C}_{2} \mathrm{O}_{4}, 33^{\circ} \mathrm{C}$ ) a satisfactorily high level of G11 activity and low level of F10 activity could be realized. The resulting xylanase preparation was more favorable for xylo-oligosaccharide production than enzymes prepared under other culture conditions.

Xylan was the best carbon source for the transcription of the two types of xylanase. Xylose could be utilized for inducing transcription of the enzymes; however, it was less efficient in supporting the accumulation of mycelium and active enzymes (Table 3). This result might imply that other sugars released from xylan due to the hydrolysis could also be efficiently metabolized by this fungus. Furthermore, the contribution from other xylan components to the accumulation of mycelium and xylanase activity were quite obvious, since the corresponding values using xylan at each time point (40h or $72 \mathrm{~h}$ ) were higher than those of using the monosaccharide xylose.

Since more significant changes in transcript abundance were observed for G11 (Fig. 1, 2, 4) in each analysis, we deduced that, 
463

464

465

466

467

468

469

470

471

472

473

474

475

the utilization of xylan was more dependent on the increased level of G11. The distinct properties of F10 and G11, in combination with the results shown in Fig. 1, 2, 4, enables us to propose a possible pathway by which xylan was biodegraded and utilized by fungus A.niger A09 (Fig. 5). Although transcript abundance of F10 and G11 can be significantly induced by some carbon sources (e.g. the sugars tested in this study), there is some background expression (stage 1, Fig. 5). This low level expression is independent of the availability of xylan (or other functionally or structurally similar compounds). The low abundance of F10 protein acts as a sensor to probe the availability of inducers. Why consider F10 to be the sensor protein? This question can be answered based on its structural features. F10 is a larger protein usually containing the carbohydrate binding module (CBM), making it a sensitive probe for inducer compounds in the surrounding environment. The strong affinity of CBM for corresponding substances makes it possible to sense the presence of inducers, even if they are at very low abundance. At the same time, from Fig. 1, we could also see that there was a rapid response of F10 to the availability of inducers like xylan, xylose, and other sugars.

As protein F10 probed and detected the inducer compounds (like xylan), the catalytic domain in F10 could hydrolyze the xylan into various fragments or monosaccharide (e.g. xylose, and other sugars). Although the identity of the actual inducer of increased expression of xylanase is still an open question (xylan fragment or xylose?), the results in Fig. 1, at least, showed that xylose could efficiently induce increased transcription levels for F10 and G11. Hence xylose is more likely the actual inducer, as suggested by de Vries et al. (1999). When xylan is present, the sensor protein F10 is capable of making more xylose quickly and efficiently because of its high reaction speed and product feature 
(predominantly monosaccharide/low DP oligosaccharides) in catalysis.

Therefore, we propose the biodegradation process of xylan by A.niger A09 is as follows. When xylan is absent, F10 and G11 are produced due to the low level of background gene expression. The F10 protein senses the existence of xylan, and when xylan is present, F10 binds to xylan and hydrolyzes it into fragments, as well as other monosaccharides. Xylose is then transported into the cell, leading to induced transcription of F10 and G11 to produce more enzymes. At the same time, xylose and other monosaccharides are also metabolized by the fungus to support its growth. This helps explain why a better growth can be witnessed when xylan is the carbon source. This would also help explain our failure in finding the conditions favoring the high production of F10 and the low production of G11.

\section{Acknowledgement}

This study was financially supported by the Special Fund for Agro-Scientific Research in the Public Interest from the Ministry of Agriculture, China (No. 201503134), and by the Program for Innovative Research Team (in Science and Technology) in University of Henan Province (No. 15IRTSTHN014). We thank Dr. Barry Flinn for his critical modification to the manuscript.

\section{Conflict of interest}

The authors declare that there is no conflict of interest with regard to the publication of this manuscript.

\section{References}

Adsul, M.G., Bastawde, K.B., and Gokhale, D.V. 2009. 
Biochemical characterization of two xylanases from yeast Pseudozyma hubeiensis producing only xylooligosaccharides. Bioresour Technol 100:6488-6495. doi: 10.1016/j.biortech.2009.07.064

Ahmed, S., Riaz, S., and Jamil, A. 2009. Molecular cloning of fungal xylanases: an overview. Appl Microbiol Biotechnol 84:19-35. doi: 10.1007/s00253-009-2079-4

Biely, P., Vrsanska, M., Tenkanen, M., and Kluepfel, D. 1997. Endo-beta-xylanases families : differences in catalytic properties. J Biotechnol 57:151-166

Bragatto, J., Segato, F., and Squina, F.M. 2013. Production of xylooligosaccharides (XOS) from delignified sugarcane bagasse by peroxide-HAc process using recombinant xylanase from Bacillus subtilis. Ind Crops Prod $51: 123-129$.

Buchert, J., Tenkanen, M., Kantelinen, A., and Viikari, L. 1994. Application of xylanases in the pulp and paper industry. Biores Technol 50:65-72

Chen, P., and Buller, C.S. 1995. Activity staining of xylanases in polyacrylamide gels containing xylan. Anal Biochem 
550 Collins, T., Gerday, C., and Feller, G. 2005. Xylanases, xylanase families and extremophilic xylanases. FEMS Microbiol Rev $29: 3-23$.

553 de Oliveira, J.M.P.F., van Passel, M.W.J., Schaap, P.J., and de

554

555

556

557

558

559

560

561

562

563

564

565

566

567

568 Graaff, L.H. 2011. Proteomic analysis of the secretory response of Aspergillus niger to D-maltose and D-xylose. PloS One 6:e20865. doi: 10.1371/journal.pone.0020865

de Souza, W.R., Maitan-Alfenas, G.P., de Gouvêa, P.F., Brown, N.A., Savoldi, M., Battaglia, E., et al. 2013. The influence of Aspergillus niger transcription factors AraR and XlnR in the gene expression during growth in D-xylose, L-arabinose and steam-exploded sugarcane bagasse. Fungal Genet Biol FG B 60:29-45. doi: 10.1016/j.fgb.2013.07.007

Deutschmann, R., and Dekker, R.F.H. 2012. From plant biomass to bio-based chemicals: latest developments in xylan research. Biotechnol Adv 30:1627-1640. doi: 10.1016/j.biotechadv.2012.07.001

de Vries, R.P., Visser, J., and de Graaff, L.H. 1999. CreA 
569

570

571

572

573

574

575

576

577

578

579

580

581

582

583

584

585

586

587

588

modulates the XlnR-induced expression on xylose of Aspergillus niger genes involved in xylan degradation. Res Microbiol 150:281-285

Gusakov, A.V., Kondratyeva, E.G., and Sinitsyn, A.P. 2011. Comparison of two methods for assaying reducing sugars in the determination of carbohydrase activities. Int J Anal Chem 2011:e283658. doi: 10.1155/2011/283658

Han, Y., and Lei, X.G. 1999. Role of glycosylation in the functional expression of an Aspergillus niger phytase (phyA) in Pichia pastoris. Arch Biochem Biophys $364: 83-90$

Harris, P.V., Xu, F., Kreel, N.E., Kang, C., and Fukuyama, S. 2014. New enzyme insights drive advances in commercial ethanol production. Curr Opin Chem Biol 19:162-170. doi: 10.1016/j.cbpa.2014.02.015

Henrissat, B. 1991. A classification of glycosyl hydrolases based on amino acid sequence similarities. Biochem J $280(\mathrm{Pt}$ 2):309-316.

Henrissat, B., and Bairoch, A. 1993. New families in the classification of glycosyl hydrolases based on amino acid 
590 Ho, W., and Ko, W. 1997. A simple method for obtaining single-spore of fungi. Bot Bull Acad Sin 38:41-44.

592 Jeffries, T.W. 1996. Biochemistry and genetics of microbial xylanases. Curr Opin Biotech 7:337-342.

594

595

596

597

598

599

600

601

602

603

604

605

606

607

608

Kniemeyer, O. 2011. Proteomics of eukaryotic microorganisms: The medically and biotechnologically important fungal genus Aspergillus. Proteomics 11:3232-3243. doi: 10.1002/pmic.201100087

Leber, T.M., and Balkwill, F.R. 1997. Zymography: a single-step staining method for quantitation of proteolytic activity on substrate gels. Anal Biochem 249:24-28. doi: 10.1006/abio.1997.2170

Lee, R.C., Hrmova, M., and Burton, R.A. 2003. Bifunctional family 3 glycoside hydrolases from barley with alpha-L-arabinofuranosidase and beta-D-xylosidase activity characterization, primary structure and COOH-terminal processing. J Biol Chem 278:5377-5388.

Liu, M.Q., and Liu, G.F. 2008. Expression of recombinant Bacillus licheniformis xylanase A in Pichia pastoris and 
609

610

611

612

613

614

615

616

617

618

619

620

621

622

623

624

625

626

627

628 xylooligosaccharides released from xylans by it. Protein Expr Purif 57:101-107

Olukosi, O.A., Bedford, M.R., and Adeola, O. 2007. Xylanase in diets for growing pigs and broiler chicks. Can J Anim Sci 87:227-235. doi: 10.4141/CJAS06005

Qu, W., Xue, Y., and Ding, Q. 2015. Display of fungi xylanase on Escherichia coli cell surface and use of the enzyme in xylan biodegradation. Curr Microbiol 70:779-785. doi: $10.1007 / \mathrm{s} 00284-015-0781-2$

Rivers, D.B., Gracheck, S.J., Woodford, L.C., and Emert, G.H. 1984. Limitations of the DNS assay for reducing sugars from saccharified lignocellulosics. Biotechnol Bioeng 26:800-802. doi: 10.1002/bit.260260727

Schmittgen, T.D., and Livak, K.J. 2008. Analyzing real-time PCR data by the comparative C(T) method. Nat Protoc 3:1101-1108. doi: 10.1038/nprot.2008.73

Schuster, E., Dunn-Coleman, N., Frisvad, J.C., and van Dijck, P.W.M. 2002. On the safety of Aspergillus niger-a review. Appl Microbiol Biotechnol 59:426-435. doi: $10.1007 / \mathrm{s} 00253-002-1032-6$ 
629 Wacker, M.J., and Godard, M.P. 2005. Analysis of one-step and 630 two-step real-time RT-PCR using SuperScript III. J Biomol 631 Tech JBT 16:266-271.

632 Wang, Q., Du, W., Weng, X.Y., Liu, M.Q., Wang, J.K., and Liu, 633 J.X. 2015. Recombination of thermo-alkalistable, high 634 xylooligosaccharides producing endo-xylanase from 635 Thermobifida fusca and expression in Pichia pastoris. Appl 636 Biochem Biotechnol 175:1318-1329. doi: $637 \quad 10.1007 / \mathrm{s} 12010-014-1355^{-7}$

638

639

640

641

642

643

644

645

646

647

648

649

650

651

652

653

654

655 
656

657

658

659

660

661

662

663

664

665

666

667

668

669

670

671

672

673

674

675

676

677

678

679

680

681

682

683

684

685

686

687

688

Figure captions

Fig. 1 Effect of carbon source on xylanase transcription levels revealed by real-time PCR analysis

Transcription levels were calculated using the $2^{-\Delta \Delta \mathrm{Ct}}$ method.

Values smaller than 0 mean the transcription levels were decreased by the indicated fold-number compared with the reference (the value for $\mathrm{F} 10$ at $40 \mathrm{~h}$ for samples collected from culture broth with glucose as carbon source). F10-40h stands for the level of F10 at $40 \mathrm{~h}$.

Fig. 2 Effect of nitrogen source on xylanase transcription levels revealed by real-time PCR analysis

Transcription levels were calculated using the $2^{-\Delta \Delta \mathrm{Ct}}$ method. Values smaller than 0 mean the transcription levels were decreased by the indicated fold-number compared with the reference (the value of F10 at 40h for samples collected from culture broth with yeast extract as nitrogen source). F10-40h stands for the level of F10 at 40h.

Fig. 3 The changes of mycelium weight, activity and specific activity during the culture process

Mycelium weight exhibited a continual increase, while activity and specific activity reached the maximum at $60 \mathrm{~h}$.

Fig. 4 Xylanase transcription levels revealed by real-time PCR analysis during the culture process Transcription levels were calculated using the $2^{-\Delta \Delta \mathrm{Ct}}$ method with the value of $\mathrm{F} 10$ at $40 \mathrm{~h}$ for samples collected from culture broth with xylan as carbon source, and $\left(\mathrm{NH}_{4}\right)_{2} \mathrm{C}_{2} \mathrm{O}_{4}$ as nitrogen source. F10-40h stands for the level of F10 at 40h.

Fig. 5 The proposed scheme of xylan biodegradation by A.niger 


\begin{abstract}
689 A09
690 Background level expression product F10 and G11 were secreted

691 into the medium. F10 functions as a sensor to probe for the

692 existence of the inducer xylan (or other inducers). F10 binds to

693 xylan and hydrolyzes it to produce xylose. Xylose enters the cell, 694 inducing and increased level of F10 and G11 gene expression. In

695 the regions containing the genes for F10 and G11, black

696 represent the introns, and white the exons.
\end{abstract}




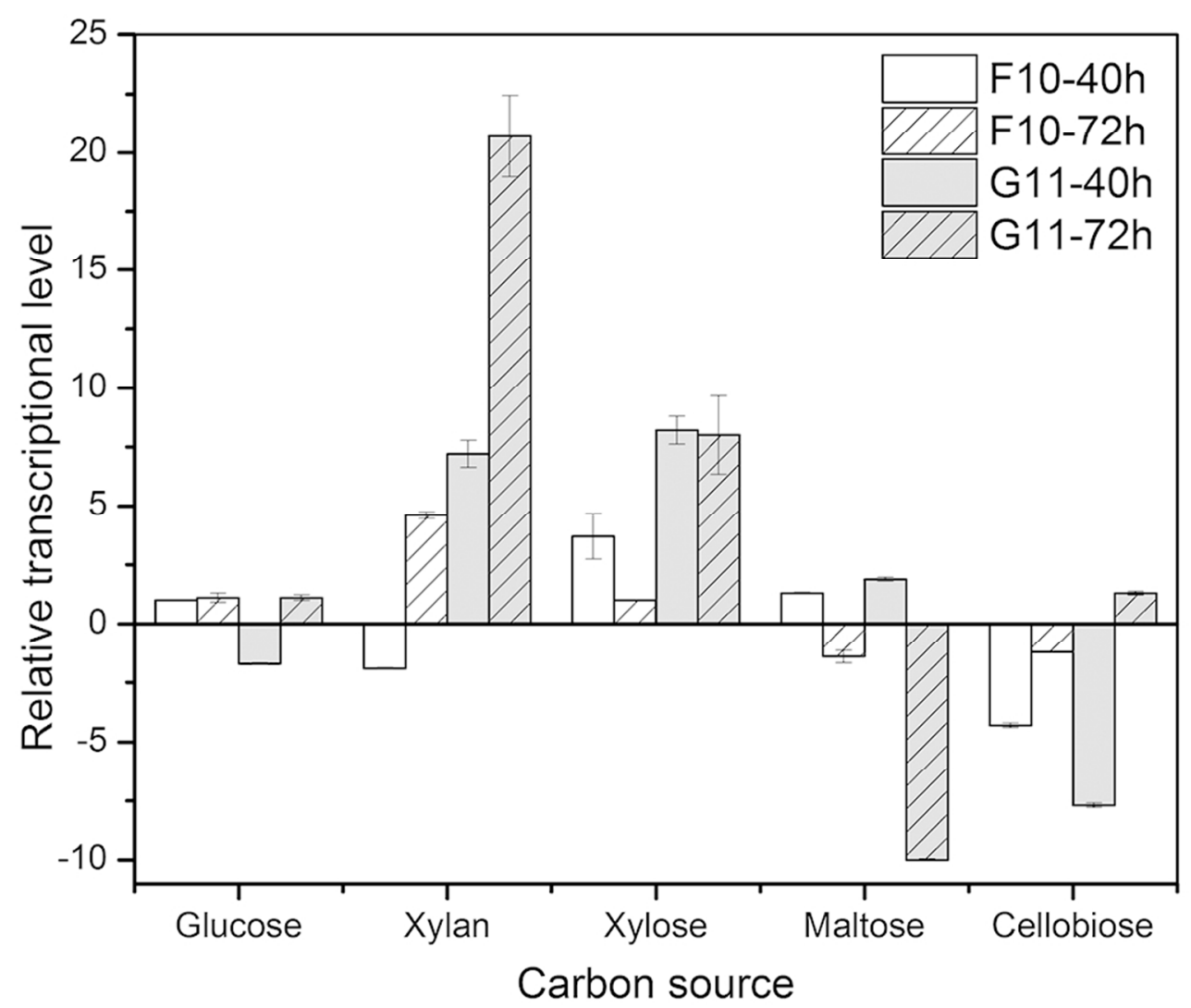

Fig. 1 Effect of carbon source on xylanase transcription levels revealed by real-time PCR analysis Transcription levels were calculated using the $2-\Delta \Delta \mathrm{Ct}$ method. Values smaller than 0 mean the transcription levels were decreased by the indicated fold-number compared with the reference (the value for F10 at $40 \mathrm{~h}$ for samples collected from culture broth with glucose as carbon source). F10-40h stands for the level of F10 at $40 \mathrm{~h}$.

$83 \times 71 \mathrm{~mm}(300 \times 300$ DPI $)$ 


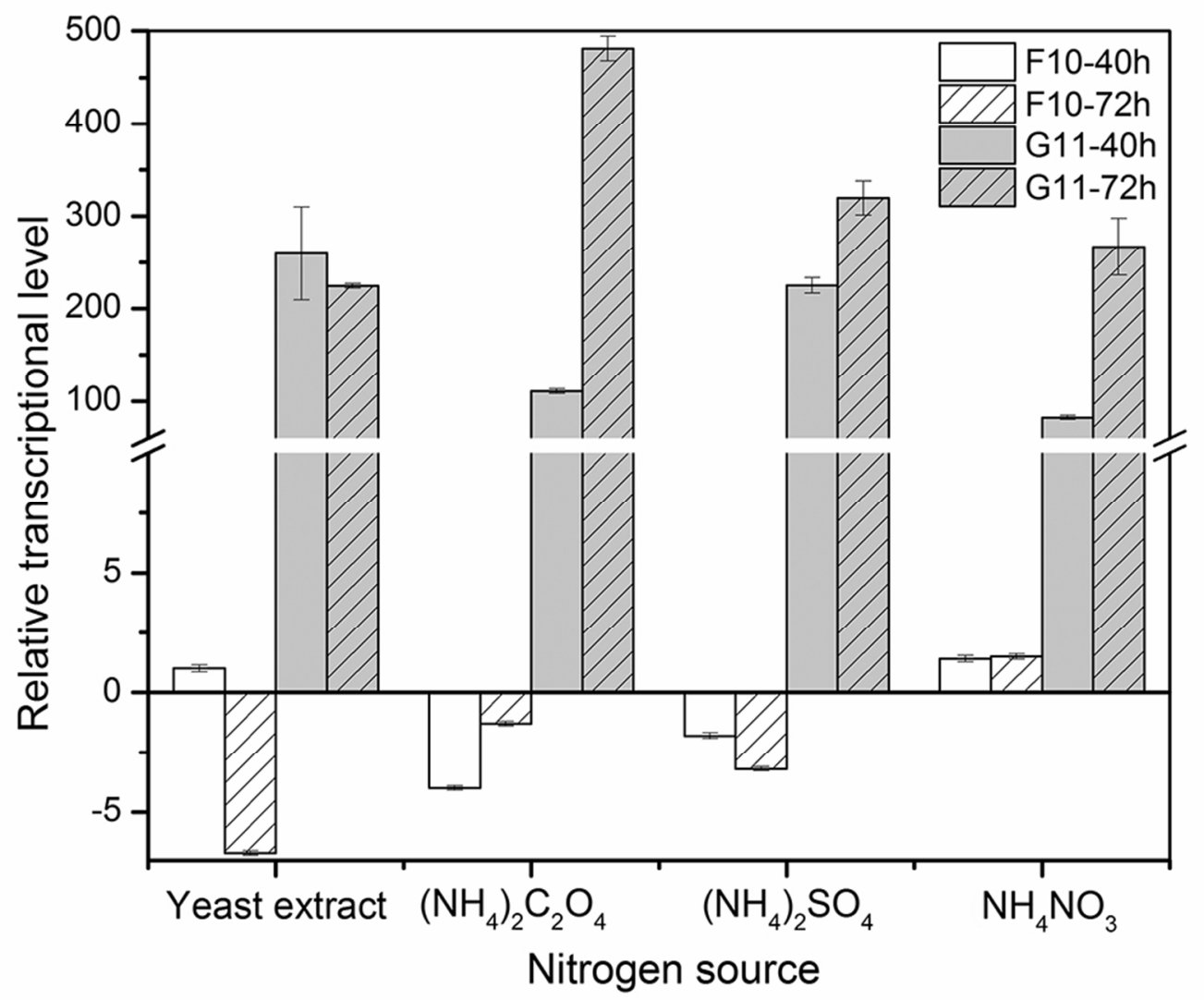

Fig. 2 Effect of nitrogen source on xylanase transcription levels revealed by real-time PCR analysis Transcription levels were calculated using the $2-\Delta \Delta \mathrm{Ct}$ method. Values smaller than 0 mean the transcription levels were decreased by the indicated fold-number compared with the reference (the value of F10 at $40 \mathrm{~h}$ for samples collected from culture broth with yeast extract as nitrogen source). F10-40h stands for the level of $\mathrm{F} 10$ at $40 \mathrm{~h}$.

$83 \times 70 \mathrm{~mm}(300 \times 300$ DPI $)$ 


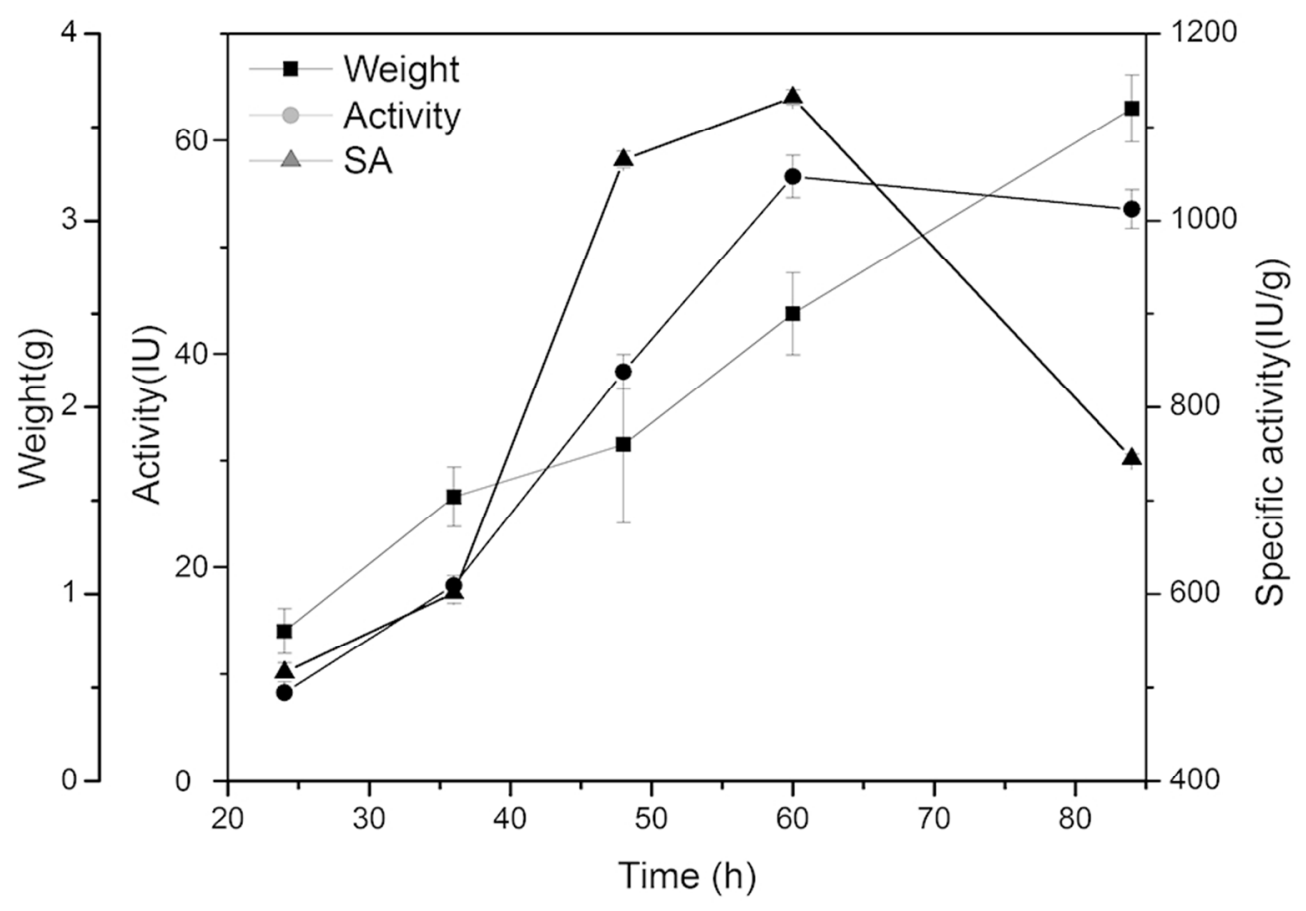

Fig. 3 The changes of mycelium weight, activity and specific activity during the culture process Mycelium weight exhibited a continual increase, while activity and specific activity reached the maximum at $60 \mathrm{~h}$.

$83 \times 58 \mathrm{~mm}(300 \times 300 \mathrm{DPI})$ 


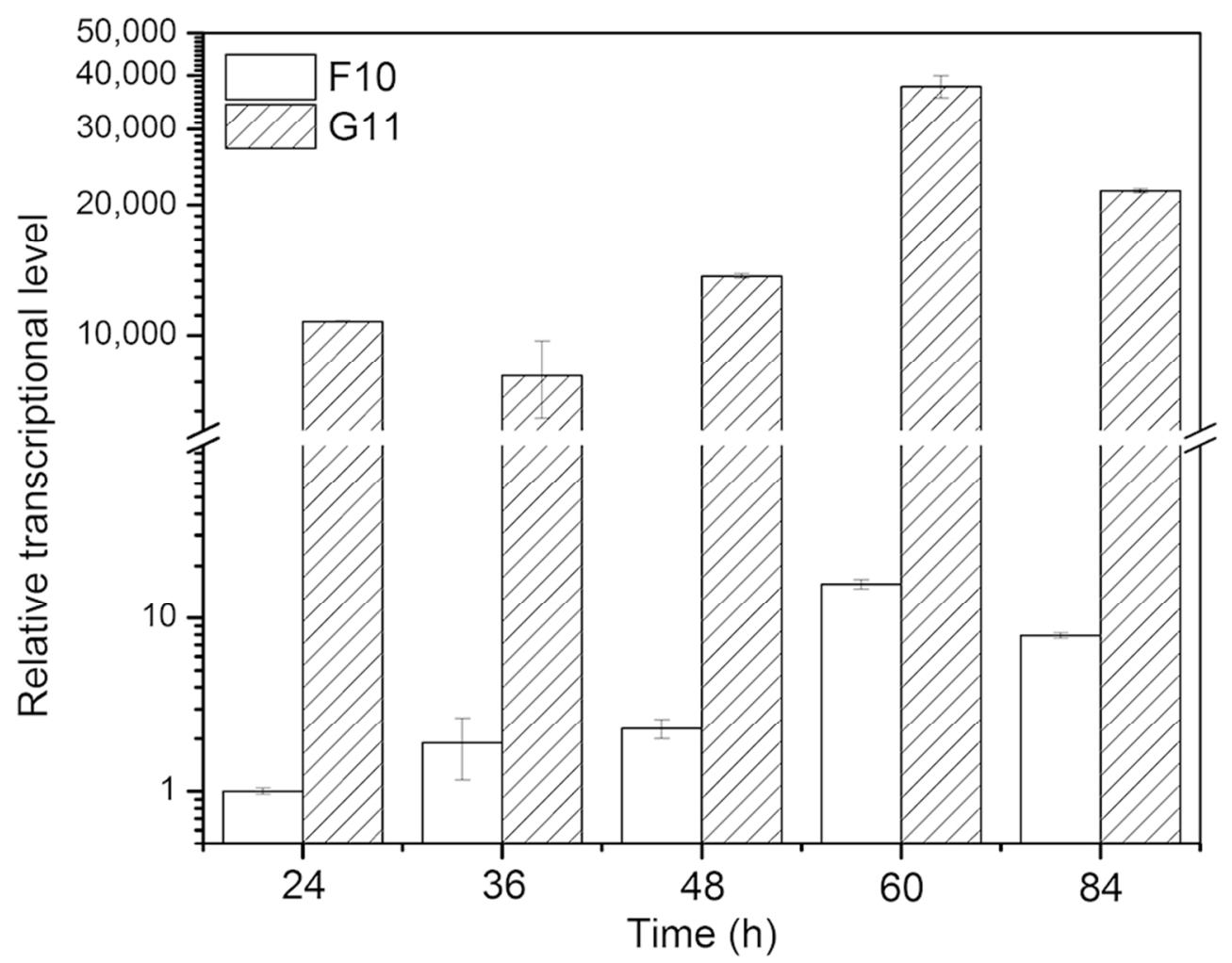

Fig. 4 Xylanase transcription levels revealed by real-time PCR analysis during the culture process Transcription levels were calculated using the $2-\Delta \Delta \mathrm{Ct}$ method with the value of $\mathrm{F} 10$ at $40 \mathrm{~h}$ for samples collected from culture broth with xylan as carbon source, and (NH4)2C2O4 as nitrogen source. F10-40h stands for the level of $\mathrm{F} 10$ at $40 \mathrm{~h}$. $83 \times 65 \mathrm{~mm}(300 \times 300 \mathrm{DPI})$ 


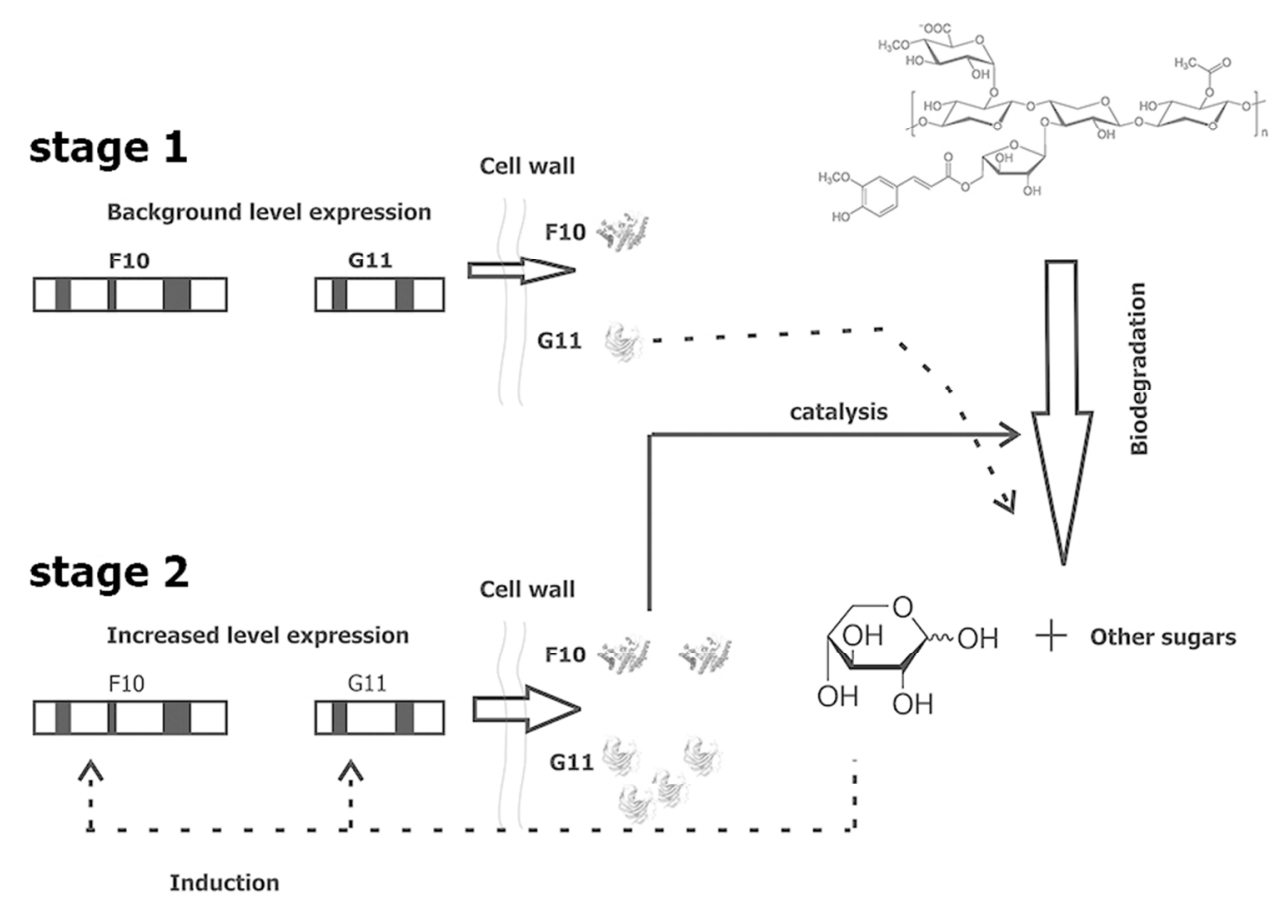

Fig. 5 The proposed scheme of xylan biodegradation by A.niger A09

Background level expression product F10 and G11 were secreted into the medium. F10 functions as a sensor to probe for the existence of the inducer xylan (or other inducers). F10 binds to xylan and hydrolyzes it to produce xylose. Xylose enters the cell, inducing and increased level of F10 and G11 gene expression. In the regions containing the genes for $\mathrm{F} 10$ and $\mathrm{G} 11$, black represent the introns, and white the exons. 


\section{Tables}

Table 1. Primers used in this study.

\begin{tabular}{ll}
\hline Primers & Sequence(5'->3') \\
\hline 18SrRNAF & ACTCACCAGGTCCAGACAAAATAAG \\
18SrRNAR & AAGCAGACAAATCACTCCACCA \\
F10F & CGGACAAGAATACACTGATCGAAG \\
F10R & TTGACAACATCCCAGGCATAAA \\
G11F & GCAAAACTACAACGGCAACC \\
G11R & AGCCCAGACCAACGACAAA \\
\hline
\end{tabular}


Table 2. The effect of different carbon source on xylanase production capacity and mycelium growth of $A$. niger A09

\begin{tabular}{ccccccc}
\hline Carbon source $(2 \%)$ & \multicolumn{2}{c}{ Activity $(\mathrm{IU} / \mathrm{ml})$} & \multicolumn{2}{c}{ Weight $(\mathrm{g})$} & \multicolumn{2}{c}{ Specific activity (IU/g) } \\
\cline { 2 - 7 } & $40 \mathrm{~h}$ & $72 \mathrm{~h}$ & $40 \mathrm{~h}$ & $72 \mathrm{~h}$ & $40 \mathrm{~h}$ & $564.5 \pm 39.5$ \\
xylan & $15.3 \pm 0.2$ & $45.14 \pm 1.3$ & $3.15 \pm 0.28$ & $4 \pm 0.66$ & $243 \pm 12.1$ & $456.5 \pm 21.6$ \\
xylose & $4.21 \pm 0.8$ & $30.6 \pm 3.3$ & $1.55 \pm 0.12$ & $3.35 \pm 0.52$ & $135 \pm 15.7$ & $9 \pm 1.1$ \\
cellobiose & $0.77 \pm 0.13$ & $0.69 \pm 0.17$ & $3.67 \pm 0.37$ & $3.85 \pm 0.83$ & $10.5 \pm 2.2$ & $12.5 \pm 0.9$ \\
glucose & $0.46 \pm 0.19$ & $0.54 \pm 0.05$ & $1.35 \pm 0.1$ & $2.20 \pm 0.21$ & $17 \pm 1.9$ & $22 \pm 1.6$ \\
maltose & - & $0.92 \pm 0.16$ & $1.12 \pm 0.11$ & $2.10 \pm 0.23$ & - & - \\
lactose & - & - & - & - & - & - \\
arabinose & - & - & - & - & -
\end{tabular}

Note: Activity: xylanase activity of crude enzyme; Weight: the fresh weight of mycelium after washing; Specific activity: the ratio of the total xylanase activity in crude enzyme to the total mycelium weight. 
Table 3. The effect of different nitrogen source on xylanase production capacity and mycelium growth of $A$. niger A09

\begin{tabular}{ccccccc}
\hline \multirow{2}{*}{ Nitrogen source $(0.3 \%)$} & \multicolumn{2}{c}{ Activity (IU/ml) } & \multicolumn{2}{c}{ Weight $(\mathrm{g})$} & \multicolumn{2}{c}{ Specific activity (IU/g) } \\
\cline { 2 - 7 } & $40 \mathrm{~h}$ & $72 \mathrm{~h}$ & $40 \mathrm{~h}$ & $72 \mathrm{~h}$ & $40 \mathrm{~h}$ & $72 \mathrm{~h}$ \\
\hline Yeast extract & $38.3 \pm 0.86$ & $43.5 \pm 0.21$ & $1.8 \pm 0.2$ & $2.2 \pm 0.1$ & $1063 \pm 1.6$ & $988.6 \pm 5.8$ \\
$\left(\mathrm{NH}_{4}\right)_{2} \mathrm{C}_{2} \mathrm{O}_{4}$ & $35.8 \pm 0.24$ & $52 \pm 0.91$ & $1.8 \pm 0.1$ & $2.5 \pm 0.09$ & $993.6 \pm 1.9$ & $1040 \pm 4.7$ \\
$\left(\mathrm{NH}_{4}\right)_{2} \mathrm{SO}_{4}$ & $32.5 \pm 0.43$ & $45.3 \pm 0.39$ & $1.7 \pm 0.15$ & $2.1 \pm 0.07$ & $955.3 \pm 5.9$ & $1077.4 \pm 7.1$ \\
$\mathrm{NH}_{4} \mathrm{NO}_{3}$ & $42.2 \pm 1.1$ & $58 \pm 0.54$ & $2.1 \pm 0.2$ & $2.4 \pm 0.12$ & $1004.8 \pm 6.2$ & $1208.3 \pm 4.6$ \\
\hline
\end{tabular}

Note: Activity: xylanase activity of crude enzyme; Weight: the fresh weight of mycelium after washing; Specific activity: the ratio of the total xylanase activity in crude enzyme to the total mycelium weight. 